\title{
Essais de l'appareil "Compteur Coulter" utilisé pour la détermination du nombre de cellules totales des laits de troupeaux
}

\author{
par \\ R. GRAPPIN et R. JEUNET \\ I.N.R.A., Station Expérimentale Laitière (39) Poligny
}

\section{Introduction}

On trouve dans le lait deux types principaux de cellules : des cellules épithéliales dont le nombre dépend entre autres choses du stade de lactation et des leucocytes présents en très grand nombre lorsque l'animal est atteint de mammite. Comme le nombre de cellules totales du lait et en particulier celui des leucocytes, augmente beaucoup avec la gravité de l'infection, on a mis à profit cette observation pour rechercher les animaux mammiteux, soit au moyen de la numération directe des cellules au microscope, soit par l'emploi de tests indirects basés sur la réaction entre les cellules du lait avec une solution alcaline (épreuve de Whiteside) ou un détergent (California Mastistis Test, Brabant Mastitis Reaction).

Bien que les résultats donnés par ces dernières épreaves présentent parfois des difficultés d'interprétation, on les utilise très largement car elles donnent des indications suffisantes pour détecter les mammites ainsi que le caractère de gravité de l'infection. De plus, par rapport à la méthode microscopique, elles ont l'avantage d'être simples et peu coûteuses et peuvent faire l'objet d'une mécanisation assez poussée, comme celle qui est réalisée par exemple dans certains laboratoires hollandais (Auclair et al., 1970). Mais ces épreuves rapides sont insuffisantes pour déterminer avec précision le nombre de cellules contenues dans le lait (Schipper et al. 1968 ; Zeidler et al., 1968 b ; Read JR. et al., 1969 a). Or, il existe dans plusieurs pays, soit en fait soit à l'état de projet, des réglementations concernant la qualité hygiénique du lait qui prévoient des conséquences, financières ou autres, pour le producteur, si le lait livré à la laiterie contient un nombre élevé de cellules (plus de 500000,1 million ou 1,5 million par $\mathrm{ml}$ par exemple). Dans ce cas, le laboratoire chargé des contrôles doit disposer d'une méthode de numération suffisamment précise et objective et également peu coûteuse. C'est l'intérêt 
essentiel que présentent à cet égard les appareils automatiques de numération tels que le Compteur Coulter.

Il est bien entendu que si des numérations périodiques des cellules du lait sont faites pour contrôler la qualité hygiénique des laits, les résultats donnés par ces contrôles permettront le dépistage des troupeaux atteints de mammite et faciliteront la mise en cuvre de moyens propres à faire régresser l'infection.

Grâce à un prêt de plusieurs mois consenti par la Société Coultronics France, nous avons pu procéder à des essais de l'appareil «Compteur Coulter » dont nous donnons ici les résultats.

\section{I. - Principe du Compteur Coulter. Utilisation pour la numération des cellules du lait}

Le Compteur Coulter est un appareil qui détermine, en fonction de leurs dimensions, le nombre de particules en suspension dans un électrolyte. Comme le lait contient beaucoup d'éléments en suspension et en particulier un très grand nombre de globules gras (de 2 à $6.10^{9} / \mathrm{ml}$ ), dont la dimension est voisine de celle des cellules, il faut faire en sorte que ces globules gras n'interfèrent pas dans le comptage des cellules.

\section{A. - PREPARATION DE L'ECHANTILLON DE LAIT}

Deux techniques sont utilisées pour éliminer l'influence de la matière grasse : la centrifugation ou le traitement chimique du lait avec un émulsifiant.

Dans la technique de centrifugation utilisée par Cullen (1965), Phipps et Newbould (1966), Read JR. et al. (1967) on dilue le lait dans une solution saline et, après centrifugation, on élimine le liquide surnageant contenant le lait dilué et la matière grasse. Pour effectuer les mesures, les cellules du lait formant le culot de centrifigation sont mises en suspension dans un électrolyte.

En 1966, Tolle et al. décrivent une méthode qui permet l'élimination de la matière grasse par traitement du lait à la chaleur avec une solution d'électrolyte contenant de l'éthanol et un émulsifiant, le Witopal CO. Pour éviter la destruction des cellules au cours de la conservation et du traitement chimique du lait, celui-ci est préalablement additionné de formol à la concentration de 0,07 p. 100 et maintenu $24 \mathrm{~h}$ à température ambiante ; on peut également opérer plus rapidement en plaçant les échantillons formolés pendant $30 \mathrm{mn}$ au bain-marie à $60^{\circ} \mathrm{C}$. Pendant le traitement du lait par l'électrolyte, l'action conjuguée de l'éthanol, de l'émulsifiant et de la chaleur, entraîne une clarification du milieu par dispersion des globules gras en particules très fines, stables, rendant possible le comptage sélectif des cellules.

Dijkman et al. (1966), Phipps (1968) ainsi que Pearson et al. (1970 a) obtiennent des résultats identiques avec les deux techniques, 
mais ils préfèrent la méthode de Tolle à la méthode utilisant la centrifugation car elle est plus simple, plus rapide et peut être facilement mécanisée. Au cours de ses essais, Phipps (1968) a montré que le traitement chimique du lait entraîne une diminution de la taille moyenne des cellules.

Zeidler et al. (1968) ont apporté quelques modifications à la technique initiale en remplaçant notamment le Witopal CO par du Triton X 100 qui assure une meilleure dispersion de la matière grasse.

Avant son utilisation, l'électrolyte est filtré au travers d'une membrane de façon à renfermer moins de 100 particules/ml de la taille des cellules.

La dilution à partir de laquelle le comptage est effectué est préparée en prélevant $0,1 \mathrm{ml}$ de lait additionné de formol, auquel on ajoute $9,9 \mathrm{ml}$ de solution d'électrolyte. Les tubes bouchés contenant les dilutions sont mis au bain-marie à $80^{\circ} \mathrm{C}$ pendant $10 \mathrm{mn}$. Après refroidissement, on effectue le comptage au Compteur coulter.

\section{B. - PRINCIPE DE L'APPAREIL ET COMPTAGE}

L'appareil comprend une sonde en verre munie d'un orifice calibré (100 microns). Deux électrodes entre lesquelles passe un courant électrique (fig. 1) sont placées de chaque côté de l'orifice. La sonde étant immergée dans le liquide à analyser, le passage d'une particule dans l'ouverture modifie la résistance électrique du milieu

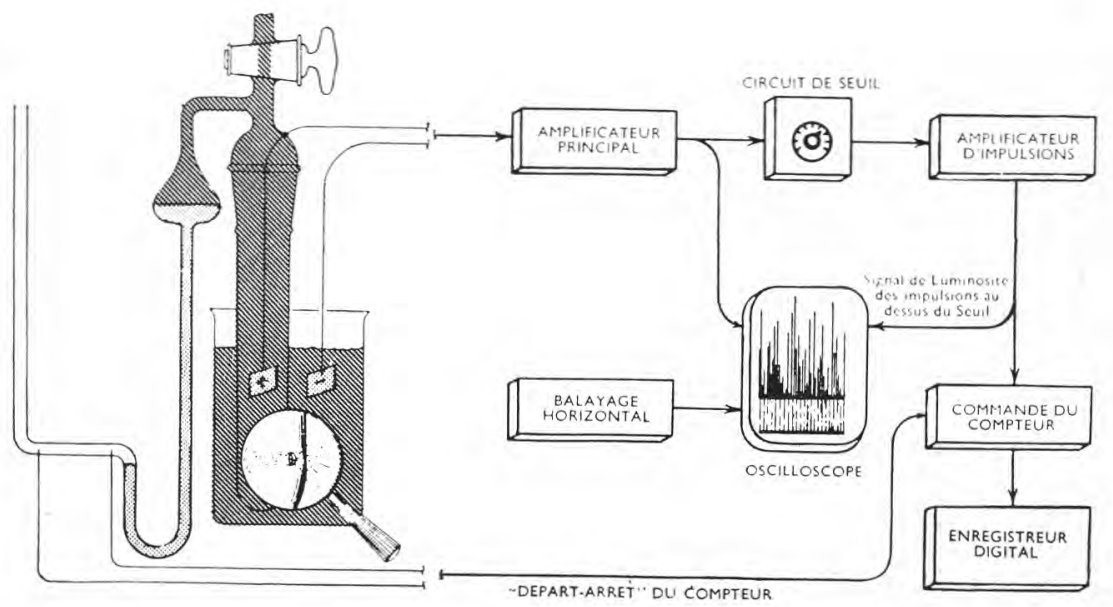

fig. 1

Schéma de fonctionnement de l'appareil Compteur Coulter (Doc. Coultronics France S.A.) 
et par conséquent l'intensité du courant entre les électrodes. Cette modification du courant se traduit par une impulsion dans un circuit approprié, impulsion dont l'intensité est proportionnelle au volume de la particule. Chaque impulsion est comptée électroniquement.

Un dispositif d'aspiration, constitué par une pompe à vide reliée à la sonde, oblige la suspension à passer par l'orifice ; en même temps, il y a déséquilibre d'un manomètre à mercure qui est relié, lui aussi, à la sonde. Lorsqu'on isole la sonde de la pompe à vide, le manomètre à mercure reprend sa position d'équilibre entraînant le passage d'un certain volume de liquide au travers de l'ouverture de la sonde. D'autre part, un dispositif spécial commande la durée du comptage correspondant au passage entre deux contacts d'un volume exactement connu de suspension.

L'appareil est également muni d'un réglage qui permet de choisir le niveau d'impulsion ou « seuil » de lecture au-dessus duquel sont effectués les comptages, ceci pour un appareil donné et dans des conditions opératoires bien déterminées. Seules les particules dont le volume dépasse le seuil sont enregistrées par le Compteur. Pour établir une relation entre les " unités de seuil » et le volume des particules qui sont comptées à chaque « seuil », on réalise un étalonnage primaire de l'appareil, à l'aide de particules dont la dimension moyenne est exactement connue (grains de latex, pollen, spores), en construisant un diagramme des fréquences du nombre des particules comptées en fonction des valeurs du seuil (fig. 2). La distribution des particules devant être normale, à la valeur de seuil correspondant à la moyenne de la distribution correspond le diamètre moyen des particules.

Il est clair qu'en raison même de son principe, le Compteur Coulter effectue le comptage de toutes les particules ayant un volume supérieur au volume du seuil choisi, que ces particules soient ou non des cellules du lait.

\section{II. - Essais de l'appareil}

\section{A. - METHODES}

1) COMPTAges microscopiques : nous avons utilisé la technique microscopique de numération de Prescott et Breed, donnant le nombre de cellules totales par $\mathrm{ml}$ de lait (NCT). Chaque échantillon de lait sert à préparer deux étalements et pour chaque étalement on effectue $2 \times 40$ lectures de champ. Le facteur de multiplication employé est de 650000 . Des sondages ont montré que la distribution des cellules par champ est conforme à une distribution de poisson.

Les étalements sont préparés à partir de laits frais.

2) Comptages électroniques : pour le traitement du lait, nous avons utilisé la technique de Tolle décrite plus haut. Les comptages 


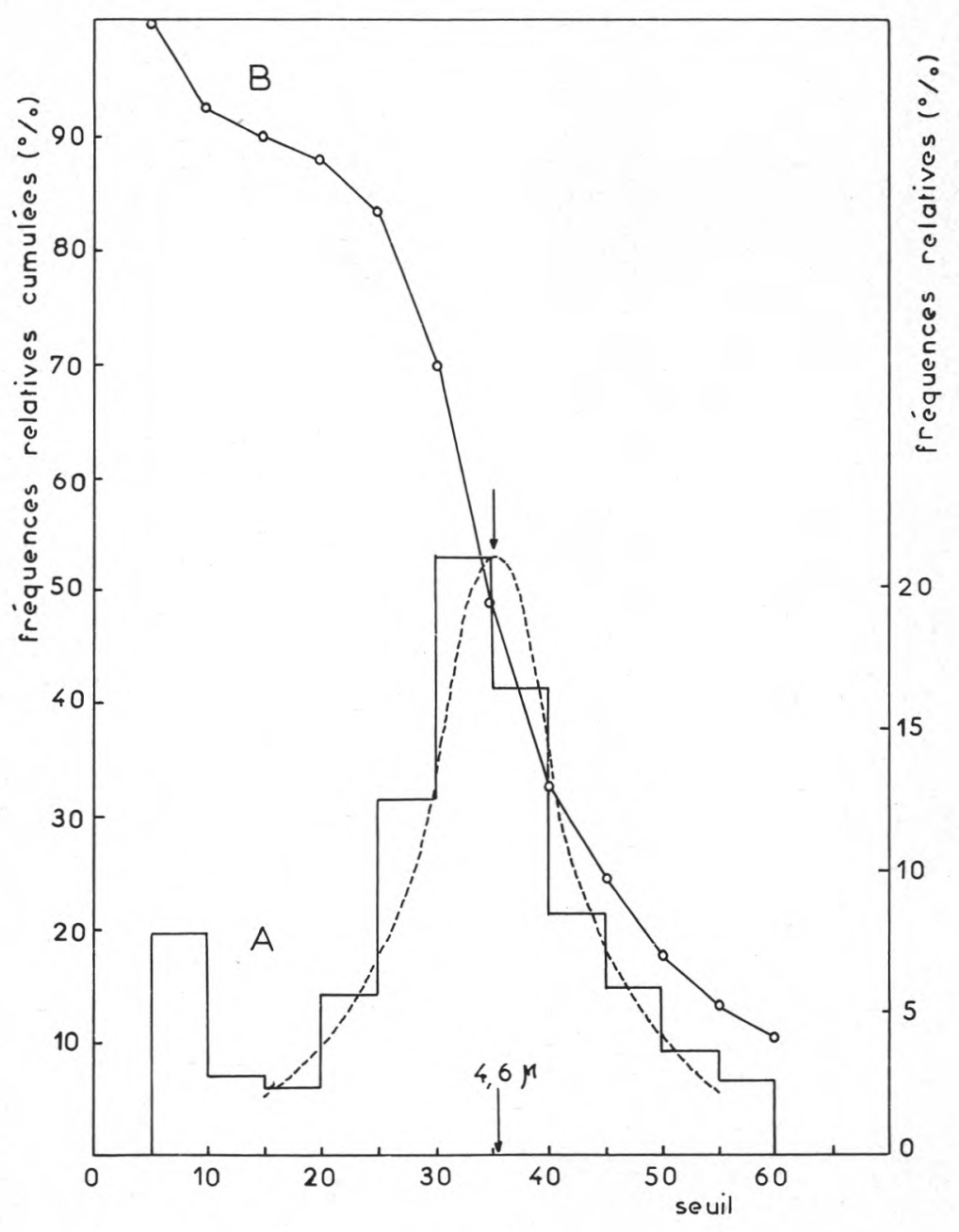

fig. 2

Distribution du nombre de particules contenues dans une suspension de particules de diamètre moyen de $4,6 \mu$, en fonction des valeurs de seuil.
A : histogramme des fréquences relatives.
B : polygone des fréquences relatives cumulées. 
sont faits à l'aide d'un Compteur Coulter modèle «A » muni d'une sonde $100 \mu$ et l'appareil est réglé pour faire des comptages sur un volume de 0,1 $\mathrm{ml}$ de suspension. La lecture directe au Compteur est multipliée par 1000 pour obtenir des résultats par ml de lait. Signalons à ce propos que, pour tenir compte de la dilution du lait par la solution de formol $(0,2 \mathrm{ml}$ d'une solution de formol à 3,5 p. 100 pour $10 \mathrm{ml}$ de lait), il convient d'ajouter $9,7 \mathrm{ml}$ de diluant au lieu de $9,9 \mathrm{ml}$.

Les lectures sur les dilutions sont toujours faites deux fois.

3) Calculs : des comptages effectués sur 1710 élevages montrent (fig. 3) que la distribution du NCT des laits est une distribution approximative log-normale. En conséquence, tous les calculs ont été faits à partir des logarithmes décimaux des nombres.
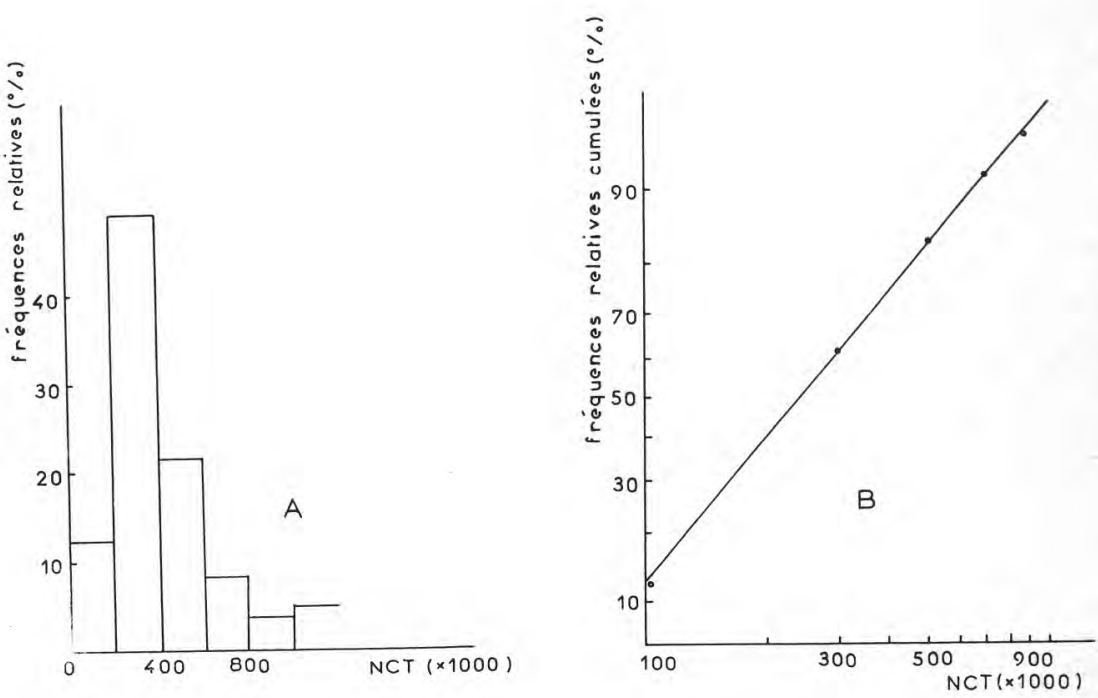

fig. 3

Distribution du nombre de cellules totales (NCT) des laits de 1710 élevages. Diagramme des fréquences relatives (A) et probit logarithmique (B).

\section{B. - PRECISION ET REPETABILITE DE LA METHODE}

1) Précision :

- Essai 1: 75 laits d'étables, prélevés au cours du dernier trimestre 1969 , renfermant entre $10^{5}$ et $2,8.10^{\circ}$ cellules par $\mathrm{ml}$, ont fait l'objet de numérations, d'une part au microscope et d'autre part au 
Compteur Coulter. Les comptages électroniques ont porté sur 3 dilutions par échantillon.

La détermination exacte du seuil de lecture nécessitant au préalable l'étude de la précision de l'appareil, nous avons choisi, pour cet essai, un seuil de lecture pour lequel les comptages microscopiques effectués sur quelques laits se sont rapprochés le plus des comptages électroniques, soit un seuil correspondant à un diamètre de $4,3 \mu$ (seuil de 30 ).

- Résultats : l'examen de la figure 4 montre que le comptage des laits ayant moins de $2.10^{5}$ cellules environ est surestimé par le Compteur Coulter, confirmant les observations de Dijkman et al. (1966, 1969), Read JR, et al. (1967), Belino et al. (1970) et Pearson et al. $(1970 \mathrm{a})$. De ce fait, nous avons éliminé ces échantillons pour ne garder, dans le calcul, que les laits ayant plus de $2.10^{5}$ cellules $/ \mathrm{ml}$.

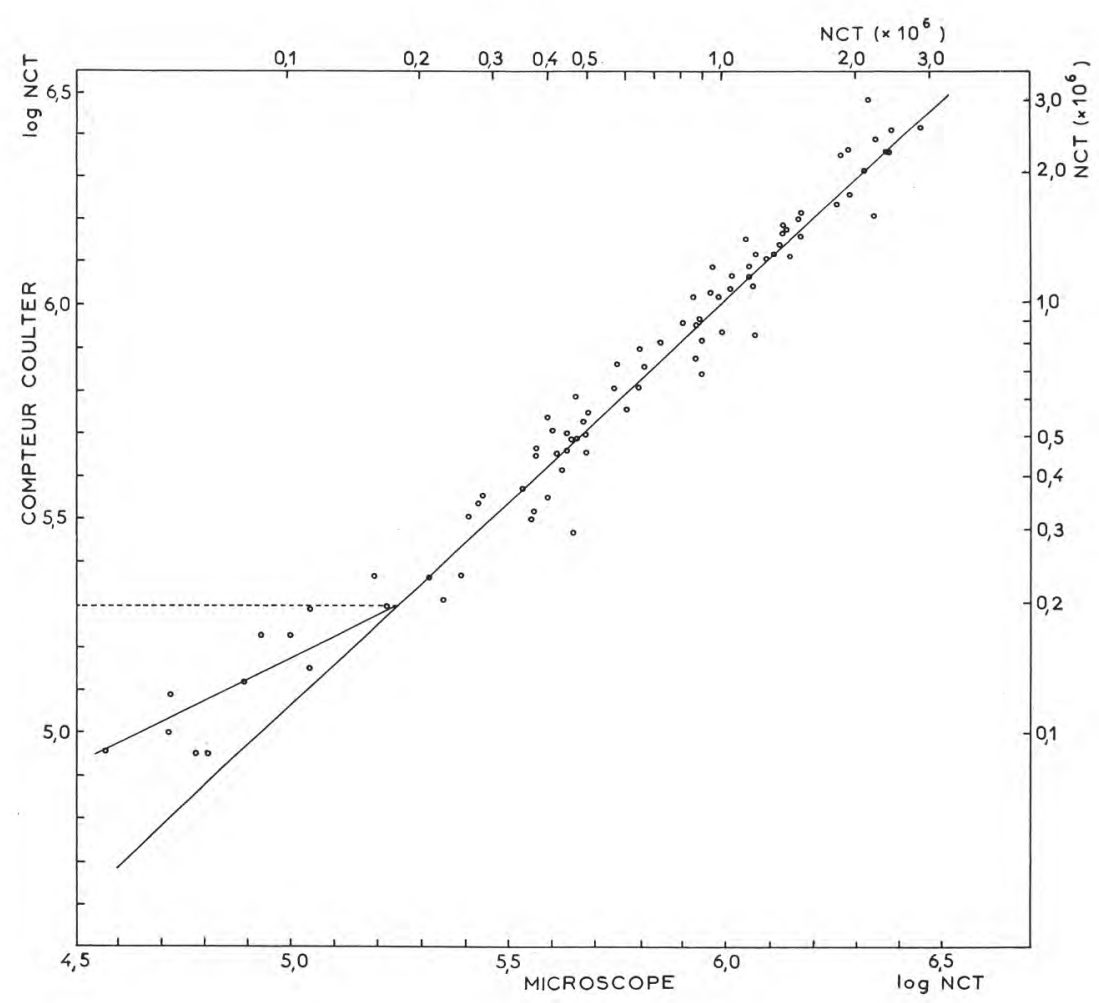

fig. 4

Relation entre les déterminations du nombre de cellules totales (NCT) de 75 laits de troupeaux, faites au microscope (x) et au Compteur Coulter (y). 
D'ailleurs, sur le plan pratique, la détermination exacte du nombre de cellules de lait ayant moins de $2.10^{5}$ cellules / $\mathrm{ml}$ présente peu d'intérêt car ces laits ne renferment pas, en général, de germes de mammite.

Calculé à partir des moyennes des comptages microscopiques et électroniques effectués sur chaque lait, le coefficient de corrélation entre les deux méthodes est de 0,976 avec un intervalle de confiance à 95 p. 100 compris entre 0,962 et 0,985 . L'écart-type résiduel s'élève à 0,0663 , soit 16,5 p. 100 si on l'exprime par rapport au nombre de cellules comptées. Entre le Compteur Coulter et la méthode microscopique, Tolle (1966), Mitchell (1967) et Pearson et at. (1970 a) obtiennent des corrélations qui varient entre 0,97 et 0,98 . Exprimée par l'écart-type des valeurs estimées, la précision du compteur trouvée par Read JR. et al. (1969 a) est voisine de 0,050 (en logarithmes décimaux), quant à Dijkman et al. (1969), ils ne donnent pas de résultats chiffrés, mais leur graphique semble indiquer un écart-type résiduel relativement élevé.

2) RÉPÉTABILITÉ :

- Essai 2: Treize laits d'étables renfermant entre $2.10^{5}$ et $1,5.10^{\circ}$ cellules/ml ont été analysés sur 12 prélèvements (soit 12 dilutions) et chaque dilution a été lue deux fois au compteur.

- Résultats : L'analyse de variance effectuée sur les logarithmes des nombres donne, pour les variations entre lectures (intradilution) et entre dilutions (intra-lait), des écarts-types respectifs de 0,0150 et 0,0091 , soit des coefficients de variation de 3,5 p. 100 et 2,1 p. 100 (tab. 1). En regroupant ces deux sources d'erreur, on

\section{TABLEAU 1}

Analyse de la variance de 13 (laits) $\times 12$ (dilutions) $\times 2$ (lectures) numérations au Compteur Coulter (essai 2)

\begin{tabular}{c|c|c|c}
\hline $\begin{array}{c}\text { Origine de la } \\
\text { variation }\end{array}$ & $\begin{array}{c}\text { Somme des } \\
\text { carrés }\end{array}$ & $\begin{array}{c}\text { Degré de } \\
\text { liberté }\end{array}$ & Carré moyen \\
\cline { 2 - 3 } $\begin{array}{c}\text { Laits } \\
\begin{array}{c}\text { Dilutions } \\
\text { (intra-lait) }\end{array}\end{array}$ & 12,83990 & 12 & 1,06999 \\
$\begin{array}{c}\text { Lectures } \\
\text { (intra-dilutions) }\end{array}$ & 0,05733 & 143 & 0,000400 \\
Totale & 12,93261 & 156 & 0,000226 \\
\hline
\end{tabular}


obtient une répétabilité de l'ordre de 4,1 p. 100 , soit un intervalle de variation des écarts entre deux déterminations sur un même échantillon de lait voisin de 16 p. 100 , dans 95 p. 100 des cas.

Read JR. et al. (1969 a) et Dijkman (1969) trouvent respectivement comme reproductibilité entre dilutions, des écarts-types de 0,012 et 0,0327 , soit des coefficients de variation de 2,8 p. 100 et 7,8 p. 100. Quant à Pearson et al. (1970 a), ils obtiennent une très bonne répétabilité des résultats, avec des coefficients de variation allant de 2,5 p. 100 à 0,9 p. 100 lorsque le nombre de cellules du lait passe de $4.10^{5}$ à $1,4.10^{6} / \mathrm{ml}$.

\section{C. - ETALONNAGE DE L'APPAREIL : CHOIX DU SEUIL DE LECTURE}

Suivant Zeidler et al. (1968 a), on détermine le seuil de lecture en construisant un diagramme cumulé du nombre de particules contenues dans un lait ayant un nombre élevé de cellules, en fonction des valeurs de seuil (fig. 5). Pour les valeurs croissantes de seuil, la courbe décroît tout d'abord rapidement au niveau des petites particules et débris, puis elle se stabilise pour donner un "plateau » et décroît ensuite lorsqu'on atteint les volumes correspondant aux volumes des cellules du lait. Théoriquement, c'est à la valeur de seuil correspondant au milieu du plateau que doivent se faire les lectures. En fait, le choix du seuil à l'intérieur des limites du plateau est délicat car on n'obtient pas un plateau réellement horizontal, mais une portion de droite oblique (fig. 5) et d'autre part, la distribution volumique des cellules peut varier suivant la « nature » du lait et rendre encore plus incertaine la détermination de ce plateau.

Aussi, pour déterminer avec le maximum de précision le seuil de lecture, nous avons réalisé un essai (essai 3), dans lequel nous avons fait, sur un nombre élevé d'échantillons de lait de teneur en cellules variable, des comptages microscopiques de référence et des comptages électroniques. Ces derniers ont été effectués à différents seuils situés dans les limites du plateau défini plus haut, soit, d'après la figure 5 , approximativement entre 20 et 50 . On a comparé ensuite pour chaque seuil les résultats obtenus, d'une part au microscope et d'autre part avec le compteur. Théoriquement, c'est au seuil pour lequel on a, entre les deux séries de résultats, à la fois le coefficient de corrélation le plus élevé, le coefficient de régression le plus proche de 1 et des moyennes égales qu'on répond le mieux aux conditions d'étalonnage de l'appareil.

- Essai 3 : Sur 87 laits de troupeaux ayant plus de 200000 cellules $/ \mathrm{ml}$, nous avons déterminé le nombre de cellules totales par la méthode microscopique et avec le compteur. Les déterminations au compteur (1 détermination par échantillon) ont été faites aux seuils arbitraires de $23,30,44$ et 55 . 


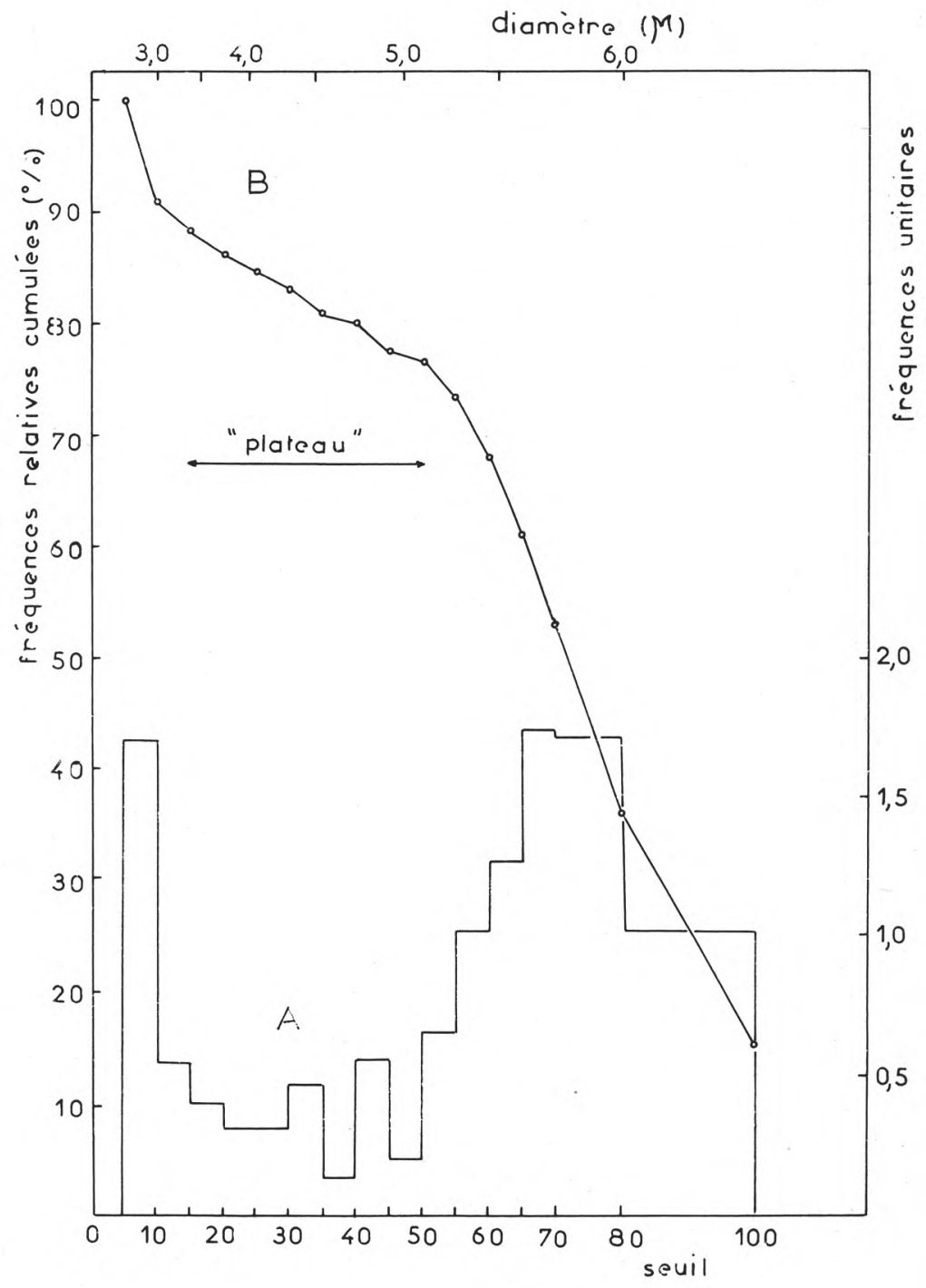

fig. 5

Distribution des fréquences du nombre de particules contenues dans un lait en fonction de leur dimension.

A : hystogramme normé.

B : polygone des fréquences relatives cumulées. 
Précision des comptages au Compteur Coulter en fonction du réglage de seuil de l'appareil (essai 3)

\begin{tabular}{|c|c|c|c|c|c|c|}
\hline \multicolumn{2}{|c|}{ Réglage } & \multirow{2}{*}{ Régression } & \multirow{2}{*}{$\mathrm{r}$} & \multirow{2}{*}{$\sigma_{\mathrm{r}}$} & \multicolumn{2}{|c|}{$\begin{array}{l}\text { Différence moyenne } \\
\text { C C - Micro }\end{array}$} \\
\hline $\begin{array}{l}\text { Valeur de } \\
\text { seuil }\end{array}$ & $\begin{array}{c}\text { Diamètre } \\
(\mu)\end{array}$ & & & & $\log$. & p. 100 \\
\hline 23 & 4,0 & $\mathrm{CC}=0,9070 . \mathrm{M}+0,6161$ & 0,971 & 0,0678 & $+0,0700$ & $+17,5$ \\
\hline 30 & 4,3 & $\mathrm{CC}=0,9510 . \mathrm{M}+0,3187$ & 0,972 & 0,0692 & $+0,0309$ & $+7,4$ \\
\hline 44 & 4,9 & $\mathrm{CC}=0,9803 . \mathrm{M}+0,0750$ & 0,969 & 0,0761 & $-0,0407$ & $-9,8$ \\
\hline 55 & 5,3 & $\mathrm{CC}=0,9885 . \mathrm{M}-0,0553$ & 0,950 & 0,0979 & $-0,1229$ & $-32,7$ \\
\hline
\end{tabular}

CC: Lecture au Compteur Coulter (en logarithme).

M : Lecture au microscope (en logarithme).

$r$ : Coefficient de corrélation.

$\sigma_{\mathrm{r}}$ : Ecart-type résiduel.

p. 100 : Exprimé en p. 100 du nombre de cellules comptées. 
- Résultats : L'examen du tableau 2 montre que, parallèlement à l'augmentation du seuil, le coefficient de régression augmente et tend vers 1 et le coefficient de corrélation diminue (la valeur maximum étant située au seuil de 30). Par ailleurs, c'est au seuil de 36 (déterminé graphiquement) qu'il y a égalité entre les comptages microscopiques et électroniques. Il semble donc qu'en choisissant ce seuil de 36 , qui correspond à un diamètre de lecture de $4,6 \mu$ environ, on se place dans les meilleures conditions (corrélation élevée, pente peu différente de 1 , égalité des moyennes) pour calibrer correctement l'appareil.

\section{D. - INFLUENCE DES CONDITIONS DE CONSERVATION DU LAIT}

- Essai 4: Quinze laits de troupeaux renfermant entre 3.10 et $4,5.10^{6}$ cellules par ml sont analysés au Compteur Coulter après 0,1 , 2,3 , et $6 \mathrm{j}$ de conservation à $20^{\circ} \mathrm{C}$ avec l'un des produits de conservation suivants : $\mathrm{HCHO}(0,7$ p. 1000$), \mathrm{Hg} \mathrm{Cl}_{2}(0,7$ p. 1000$)$ et $\mathrm{K}_{2} \mathrm{Cr}_{2} \mathrm{O}_{7}$ (1 p. 1000). Une série de laits est maintenue à $4^{\circ} \mathrm{C}$ sans produit de conservation.

Si, dans cet essai, le formol est considéré essentiellement comme un produit de conservation du lait, il ne faut pas oublier qu'il joue également le rôle important de fixateur des cellules du lait, qu'il soit accompagné ou non d'un chauffage du lait, dans la technique de Tolle. Les laits conservés par le formol pourront donc être analysés directement. Par contre, les échantillons de lait conservés sans addition d'aucun conservateur, ou ceux additionnés de sublimé ou de dichromate de potassium, devront subir, avant le comptage, le traitement par le formol et le chauffage.

- Résultats : Les comparaisons des moyennes de chaque série par rapport à la série témoin (numération sur le lait frais) sont faites à l'aide du test $t$.

1) LAITS CONSERVÉS AU FROID SANS PRODUIT DE CONSERVATION. Malgré une augmentation moyenne de 0,8 à 4 p. 100 des résultats obtenus après conservation du lait (tab. 3), les différences par rapport au témoin ne sont pas significatives.

Dijkman et al. (1969), de même que Belino et al. (1970) n'ont pas noté de variation des résultats après une durée de conservation de $72 \mathrm{~h}$ et plus à $4^{\circ} \mathrm{C}$.

Par contre, sur des laits de tank de ferme âgés de 48 h, Read JR. et al. (1967 et 1969 a) trouvent une augmentation significative, de 10 p. 100 environ, après $48 \mathrm{~h}$ de conservation du lait. Quant à Phipps et Newbould (1966) ils constatent de légères variations, soit positives, soit négatives, des résultats. 
TABLEAU 3

Influence des conditions de conservation du lait sur la numération des cellules au Compteur Coulter. Différence moyenne relative par rapport au témoin (essai 4)

\begin{tabular}{|c|c|c|c|c|}
\hline \multirow{2}{*}{$\begin{array}{l}\text { Durée de } \\
\text { conservation } \\
\text { (jour) }\end{array}$} & \multicolumn{4}{|c|}{ Produit de conservation } \\
\hline & $\begin{array}{l}\text { Sans produit } \\
\text { de conservation }\end{array}$ & $\begin{array}{c}\text { HCHO } \\
\text { (sans chauffage) }\end{array}$ & $\mathrm{Hg} \mathrm{Cl}{ }_{2}$ & $\mathrm{~K}_{2} \mathrm{Cr}_{2} \mathrm{O}_{7}$ \\
\hline 0 & témoin & $-26 \%$ & $+\underset{(*)}{3,1 \%}$ & $+\underset{(*)}{3,9 \%}$ \\
\hline 1 & $+2,7 \%$ & $+1,2 \%$ & $\begin{array}{c}+\underset{(* *}{7,3} \% \\
\left.{ }^{* *}\right)\end{array}$ & $+\underset{(*)}{5,0 \%}$ \\
\hline 2 & $+3,4 \%$ & $+2,3 \%$ & $+\underset{(* *)}{9,7 \%}$ & $+2,9 \%$ \\
\hline 3 & $+4,0 \%$ & $+\underset{(*)}{6,5 \%}$ & $+\underset{(* *)}{11,4 \%}$ & $+5,9 \%$ \\
\hline 6 & $+0,8 \%$ & $+\underset{(* *)}{12,1 \%}$ & $+\underset{(* *)}{9,4 \%}$ & $+1,9 \%$ \\
\hline
\end{tabular}

*: significatif au seuil de 0,05 .

**: significatif au seuil de 0,01 . 
2) LAITS CONSERVÉS AVEC DU FORMOL : Les numérations faites quelques heures seulement après l'addition du formol donnent des résultats très inférieurs (-26 p. 100) au témoin et confirment que, sans le chauffage, il faut attendre $24 \mathrm{~h}$ avant de faire les numérations.

Après 3 ou $4 \mathrm{j}$ de conservation, on observe une augmentation significative des résultats, déjà signalée par Phipps (1968) (tab. 3).

3) LAITS CONSERVÉS AVEC DU SUBLIMÉ OU DU DICHROMATE DE POTASSIUM : Il semble que la conservation du lait avec du dichromate de potassium n'entraîne pas de modifications importantes des résultats. En revanche, l'influence du sublimé est beaucoup plus significative, puisqu'on observe des différences qui vont de $+3,1$ p. 100 à $+11,4$ p. 100 (tab. 3).

Nous avons observé dans cet essai, que le sublimé et le dichromate de potassium réduisent, de façon plus ou moins accentuée suivant la durée et la température de conservation, la taille moyenne des cellules du lait. Mais cette réduction n'entraîne pas, dans les conditions expérimentales utilisées, de diminution des résultats, ceuxci étant, au contraire, toujours surestimés.

\section{III. - Prix de revient de l'analyse}

Nous nous limiterons à donner les éléments principaux permettant de calculer le coût de l'analyse.

- Cadence d'analyses : environ 1000 dosages par journée dé $8 \mathrm{~h}$ et pour deux personnes.

— Prix de l'appareil, modèle «F (décembre 1970) : 24000 F H.T.

- Produits chimiques : moins de 0,02 F par analyse.

- Il faut ajouter un filtre pour la solution d'électrolyte ( $500 \mathrm{~F}$ ), un bain-marie, ainsi que le petit matériel de laboratoire.

Signalons que la Société Coultronics France, fabrique une chaîne automatique comprenant un bloc électronique, un passeur automatique d'échantillons et une imprimante (fig. 6).

Cet ensemble, dont le prix est de $45000 \mathrm{~F} \mathrm{H.T}$. environ, permet le passage et la lecture automatiques de 100 échantillons à l'heure avec une double lecture par échantillon.

\section{IV. - Discussion}

1) La technique de préparation des échantillons de lait établie par Tolle ainsi que le comptage des cellules avec l'appareil Coulter, 
fig. 6

Schéma de la chaîne automatique de comptage électronique des cellules du lait (Doc. Coultronics France S.A.)

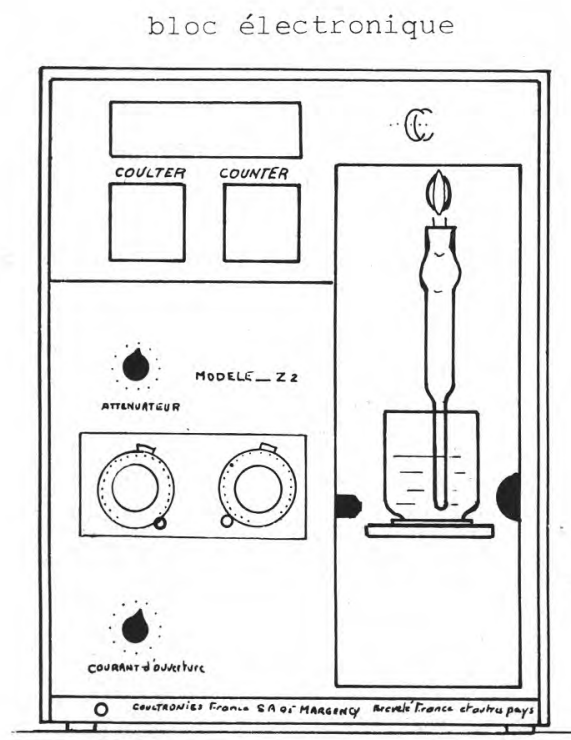

Programmation

du seuil de détection en fonction de

l'utilisation.

Bloc détection

pour d'autres applications.
Passage automatique des échantillons au niveau de la détection

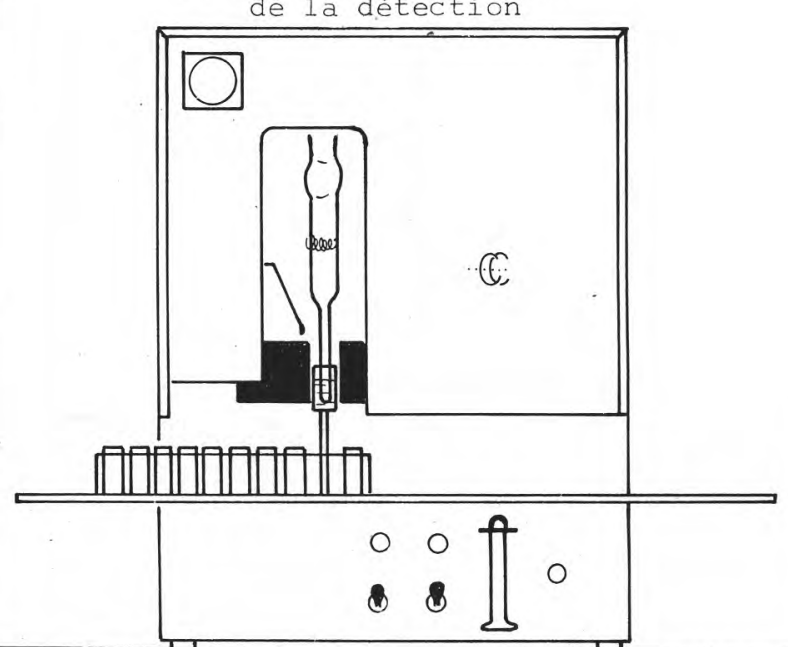

Cadence :

100 échantillons/h avec une double numération.

Sécurité :

Visualisation en permanence de I'orifice.

Alarme en cas de bouchage.
Programmateur et Imprimante

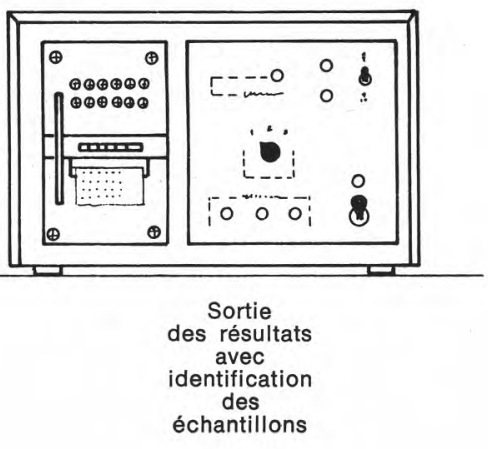


sont des opérations simples qui ne nécessitent pas de la part de l'opérateur une grande technicité. Cependant, pour obtenir de bons résultats, certaines phases de l'analyse exigent des soins et une attention toute particulière.

a) Au cours de la conservation, les cellules du lait remontent en surface avec la matière grasse. Il faut donc réchauffer l'échantillon à $35^{\circ} \mathrm{C}$ environ et opérer un mélange soigneux avant de faire le prélèvement. On peut dire que la préparation d'un échantillon de lait en vue d'une numération cellulaire demande les mêmes précautions que pour un dosage de matière grasse. Read JR. et al. (1969 b) indiquent que pour des échantillons de lait de tank de ferme (non réchauffés), la technique d'agitation est importante.

b) La vaisselle utilisée pour l'analyse (récipients, tubes, bouchons) doit être parfaitement propre et tenue à l'abri de la poussière.

c) Après le traitement du lait par la solution d'électrolyte, on peut attendre 2 ou $3 \mathrm{~h}$ avant d'effectuer les comptages ; au-delà, on observe une augmentation puis une diminution des résultats (Phipps, 1968). Mais quel que soit le temps d'attente, s'il faut mélanger soigneusement la solution avant de faire le comptage, il faut surtout éviter d'incorporer des bulles d'air par une agitation trop forte. A ce propos, il est recommandé pour chaque dilution, de faire passer le liquide dans la sonde quelques secondes avant de commencer le premier comptage pour stabiliser le flux et permettre aux bulles d'air de se disperser.

2) Nos résultats concernant la répétabilité et la précision de la méthode appellent quelques remarques. Tout d'abord, l'appareil que nous avons utilisé ne comportait pas de dispositif d'alarme en cas de bouchage de l'orifice de la sonde, comme cela est prévu sur les nouveaux appareils ; ce point est important car, s'il est facile de détecter un bouchage complet de la sonde, il est par contre beaucoup plus difficile de se rendre compte d'un bouchage partiel.

D'autre part, à la suite d'un certain nombre d'observations, nous avons apporté au cours de nos essais des améliorations sensibles au mode opératoire. Il est donc probable qu'en utilisant un appareil plus perfectionné et en bénéficiant d'une plus grande expérience on puisse obtenir de meilleurs résultats. Quoi qu'il en soit, pour des numérations précises, il est recommandé de faire au moins deux lectures par dilution et, si elles diffèrent de plus de 10 p. 100, une troisième lecture est nécessaire (Pearson et al., 1970 b).

En ce qui concerne la précision de l'appareil (essai 1), nous devons souligner que, pour réduire le plus possible les erreurs d'analyse et en particulier les erreurs de numération sous le microscope (pour lesquelles nous avons trouvé un coefficient de variation voisin de 17 p. 100), nous avons multiplié le nombre de déterminations par échantillon de lait analysé. Malgré ces précautions, il est probable que la valeur de 16,5 p. 100 de l'écart-type que nous avons indiqué 
plus haut soit surestimée, en particulier du fait de l'imprécision de la méthode de référence (numération sous le microscope). D'ailleurs, aussi bien pour la répétabilité que pour la précision, les résultats assez variables trouvés par les différents auteurs et mentionnés plus haut montrent bien l'influence prépondérante des conditions expérimentales dans lesquelles sont réalisés les essais.

3) Comme tous les appareils basés sur une méthode indirecte de mesure, le Compteur Coulter doit être étalonné par rapport à une méthode de référence, qui est ici la méthode microscopique. Mais c'est une technique délicate, peu précise et qui demande une assez grande qualification de l'opérateur. Par ailleurs, pour étalonner l'appareil, nous avons vu qu'il faut faire des mesures sur un nombre élevé de laits et que pour chaque lait, il faut répéter les mesures à des seuils différents. Cette méthode d'étalonnage est, semble-t-il, difficilement réalisable par la plupart des laboratoires. Il y a, bien entendu, la technique plus simple de Tolle (1966) qui consiste à construire, à partir d'un échantillon de lait, un diagramme des lectures faites au compteur en fonction des valeurs de seuil (fig. 5) ; mais nous avons vu que cette méthode est relativement imprécise.

D'autres solutions sont possibles : soit fixer un diamètre apparent de lecture de façon à ce que chaque utilisateur puisse déterminer le seuil de lecture à partir de l'étalonnage primaire de l'appareil effectué avec des particules standard (fig. 2) ; soit, plus simplement encore, mettre à la disposition de l'utilisateur des suspensions de particules dont le nombre, à la valeur de seuil critique, aura été déterminé au préalable et dans ce cas, un simple réglage du seuil permettant d'obtenir le nombre indiqué serait nécessaire (Pearson et al. 1970 b). En plus de leur simplicité, ces procédés auraient l'avantage d'uniformiser les mesures et par conséquent de rendre comparables les résultats obtenus par différents laboratoires.

Si on utilise la première solution, il reste évidemment à fixer le diamètre de lecture. Tolle (1966) et Zeidler (1968 a) ne donnent pas d'indications précises sur le diamètre de lecture utilisé. Par contre, parmi les auteurs utilisant la méthode de Tolle, Dijkman et al. (1968, $1969)$ et Pearson et al. (1970 a) obtiennent des corrélations élevées entre les comptages microscopiques et électroniques en utilisant respectivement des diamètres de 5 et $4,7 \mu$. D'après nos essais et pour des laits ayant plus de 200000 cellules $/ \mathrm{ml}$, c'est pour un diamètre de $4,6 \mu$ que les résultats sont les meilleurs. C'est donc probablement entre 4,5 et $5 \mu$ que devrait se situer le seuil de lecture.

Mais le fait de fixer un diamètre de lecture suppose qu'il n'y a pas de variations de la moyenne ni de la distribution du volume des cellules, en fonction de la saison ou de la nature des laits (laits individuels, laits de troupeaux) ; il suppose également que les conditions de conservation des laits sont bien définies. Nous avons signalé en 
effet que la conservation du lait avec du sublimé ou du dichromate de potassium par exemple modifie la taille des cellules ; sur un autre plan, Mitchell et al. (1967) ont obtenu des coefficients de régression entre les comptages microscopiques et électroniques qui sont différents selon que les analyses sont faites avec des laits frais ou avec des laits de tank de ferme.

En résumé, en même temps qu'on déterminera un diamètre de lecture, il faudra également fixer un certain nombre de paramètres quant à la technique d'analyse, la nature et la conservation des laits.

4) En pratique, le problème de la conservation du lait est important car il détermine les conditions de collecte et de stockage des échantillons de lait.

Dans le cas des laits conservés au froid sans produit de conservation et malgré des résultats assez contradictoires selon les auteurs et selon le type de lait utilisé, il semble qu'on puisse admettre, sans risque important d'erreur, une limite de $48 \mathrm{~h}$ de conservation. Read JR. et al. (1969 a) préconisent seulement 24 h pour les laits de tank de ferme.

Ce délai de $48 \mathrm{~h}$ peut également être admis si les laits sont additionnés de produit de conservation (formol, sublimé ou dichromate de potassium), en tenant compte cependant des restrictions indiquées à la suite de nos essais. Ce délai peut vraisemblablement être allongé si les laits sont gardés au froid. Cependant, comme nous ne possédons pas encore assez d'expérience dans ce domaine, on devra, dans un premier temps, respecter des normes assez strictes pour la conservation des laits. Signalons que pour des laits formolés, Pearson et al. (1970 b) fixent à deux semaines à $4^{\circ} \mathrm{C}$ et à une semaine à $22^{\circ} \mathrm{C}$, les limites de conservation du lait.

Nos essais ne constituent qu'une approche de ce dernier problème qui devra être approfondi, en particulier en fonction des conditions dans lesquelles se feront les déterminations par les différents laboratoires.

\section{Conclusion}

D'après nos résultats et ceux des expérimentateurs étrangers, il semble que le comptage électronique des cellules du lait est actuellement la technique la plus précise et probablement la plus économique, compte tenu des buts à atteindre.

Nous avons vu que la préparation des échantillons de lait et le comptage sont des opérations simples; cependant ces analyses ne peuvent, à notre avis, être réalisées que par des laboratoires spécialisés. D'autre part, pour uniformiser les résultats donnés par différents laboratoires, il serait souhaitable qu'à l'image de la GrandeBretagne (Pearson et al., $1970 \mathrm{~b}$ ) les laboratoires français disposent d'un guide technique précis, concernant aussi bien la méthode de 
Tolle que l'utilisation du Compteur Coulter, l'étalonnage des appareils et les conditions de conservation des laits.

\section{Rés u m é}

Une étude a été entreprise pour déterminer la valeur et les conditions d'utilisation de l'appareil "Compteur Coulter » pour la numération du nombre de cellules totales des laits de troupeaux. Les échantillons de lait ont été traités par voie chimique suivant la méthode de Tolle. La répétabilité des comptages entre dilutions (intra-lait) et entre lectures (intra-dilution) exprimée par le coefficient de variation est respectivement de 2,1 p. 100 et 3,5 p. 100 .

Sur 75 laits de troupeaux renfermant entre $2.10^{5}$ et $2,8.10^{6}$ cellules $/ \mathrm{ml}$, la corrélation entre les numérations microscopiques et électroniques est de 0,976 (avec un intervalle de confiance à 95 p. 100 compris entre 0,962 et 0,985$)$. L'écart-type résiduel, exprimé par rapport au nombre de cellules et déduit de la régression, est de 16,5 p. 100 . Les essais ont montré que l'appareil donne des résultats surestimés pour les laits ayant moins de $2.10^{5}$ cellules $/ \mathrm{ml}$. Pour les laits ayant plus de $2.10^{5}$ cellules $/ \mathrm{ml}$, c'est au seuil de lecture correspondant à un diamètre de $4,6 \mu$ que les résultats se rapprochent le plus, en moyenne, des résultats microscopiques.

Des essais de conservation du lait ont montré que les numérations peuvent être faites sur des laits conservés $48 \mathrm{~h}$ à $4^{\circ} \mathrm{C}$ (pourvu, bien entendu que ces laits ne soient pas altérés à l'origine). Les analyses semblent également possibles (avec quelques restrictions) sur les laits conservés avec $\mathrm{HCHO}$ ou additionnés de $\mathrm{Hg} \mathrm{Cl}_{2}$ ou de $\mathrm{K}_{2} \mathrm{Cr}_{2} \mathrm{O}_{7}$.

Le comptage électronique, associé à la méthode de Tolle, représente actuellement la méthode la plus précise pour contrôler le nombre de cellules des laits de ferme. Mais on doit souligner que la validité des résultats dépend en grande partie des précautions prises au cours de l'analyse. Aussi, avant d'être généralisée, cette technique devrait faire l'objet d'une normalisation.

\section{S u m m a ry}

In order to measure the number of cells in farm-milk, the value and reliability of a method associating a chemical treatment of the milk and the "Coulter Counter" has been studied.

The reproducibility (expressed as the coefficient of variation), is respectively 2,1 p. 100 and 3,5 p. 100 for the variations between dilutions (within-milk) and between readings (within-dilution).

For 75 herd milks, the cell content of which vary from $2.10^{5}$ to $2,8.10^{8} / \mathrm{ml}$, the correlation coefficient between the microscopic and electronic counts was found to be 0,976 (with a confidence interval at 95 p. 100 level situated between 0,962 and 0,985 ). The standard 
deviation of estimate (expressed as a percent of the number of cells counted) was $\pm 16,5$ p. 100 .

The electronic counts give high results for low count samples (below $2.10^{5}$ cells $/ \mathrm{ml}$ ). The best figures are obtained with a threshold value corresponding to a diameter of particles of $4,6 \mu$.

The influence of preservative and duration of storage have been studied. Good results were obtained on fresh samples kept 48 hours at $4^{\circ} \mathrm{C}$. The determinations may be carried out with milk preserved with $\mathrm{HCHO}, \mathrm{Hg} \mathrm{Cl} \mathrm{Cl}_{2}$ or $\mathrm{K}_{2} \mathrm{Cr}_{2} \mathrm{O}_{7}$ (with some limitations).

The need for good sampling and care during treatment in order to get accurate results is emphasized.

Before beeing widely used, this technique needs to be well standardized, especially as far as calibration, storage conditions, procedure for milk sample preparation and cell counting are concerned.

Reçu pour publication en janvier 1971.

\section{Références bibliographiques}

- Auclair (J.), Grappin (R.), Lapied (L.)., Mulier (M.) et Richard (J.) (1970. Le paiement du lait suivant sa qualité hygiénique et sa composition aux Pays-Bas. Rev. Lait. Franç., $\mathrm{n}^{\circ}$ 278, 511-15.

- Belino (E. O.), Mitchell (W. R.) and Newbould (F. H. S.) (1970). - Factors affecting the relationship between electronic and microscopic cell counts in bovine milk. J. Dairy Sci., 53, 719-26.

- Cullen (G. A.) (1965). - The use of Electronic Counters for determining the number of cells in milk. Vet. Rec., 77, 858.

- Dijkman (A. J.), Schipper (C. J.) and Walstra (P.) (1966). - Vorloopige Medeling over de Toepassing van de "Coulter Counter" voor de Bepaling van het Celgetal van Melk. Neth. Milk Dairy J., 20, 193-203.

- Dijkman (A.J.), Schipper (C. J.), Booy (C. J.) and Posthumus (G.) (1969), The estimation of the number of cells in farm milk. Neth. Milk Dairy J., $23,168-81$.

- Mitchell (W. R.), Newbould (F. H. S.) and Platonow (I.) (1967). - Electronic and microscopic counts on bulk-milk samples. Vet. Rec., 81, 298-99.

- Pearson (J.K. L.) (1969). - The advantages of assessing the somatic cell content of bulk-milk in the national herd. J. Soc. Dairy Technol., 22, 147-50.

- Pearson (J. K. L.), Wright (C. L.) and Greer (D. O.) (1970 a). - A study of methods for estimating the cell content of bulk-milk. J. Dairy Res., 37, 467-80.

- Pearson (J. K. L.), Wright (C. L.), Greer (D. O.), Phipps (L. W.) and Booth (J. M.) $(1970 \mathrm{~b})$. - Electronic counting of somatic cells in milk. A recommanded procedure for milk sample and cell counting with a Coulter Counter. Réf. : J. Dairy Res., 37, 465.

- Phipps (L. W.) and Newbould (F.H. S.) (1966). - Determination of leucocyte concentrations in cow's milk with a Coulter Counter. J. Dairy Res., 33, 51-64.

- Phipps (L.W.) (1968). - Electronic counting of cells in milk : examination of a chemical treatment of dispersal of fat. J. Dairy Res., 35, 295-302.

- Read JR. (R. B.), Reyers (A. L.), Bradshaw (J. G.) and Peeler (J. T.) (1967). Electronic counting of somatic cells in milk. J. Dairy Sci., 50, 669-74. 
- Read JR. (R. B.), Reyers (A. L.), Bradshaw (J. G.) and Peeler (J. T.) (1969 a). - Evaluation of seven procedures for detection of abnormal milk due to Mastitis. J. Dairy Sci., 52, 1359-66.

- ReAd JR. (R. B.) BradshaW (J. G.) and Brazis (A. R.) (1969 b). - Influence of milk sample agitation on abnormal milk test scores. J. Dairy Sci., 52, 1682-84.

- Schipper (C. J.), Dijkman (A. J.), Posthumus (G.) and Booy (C. J.) (1968). Estimation for cell count of assessment of milk quality. Officiel Org. $K$ ned. Zuivelboud, 60, 466-68. Réf. : Dairy Sci. Abstr., 1968, 2777.

- Tolle (A.), Zeidler (H.) und Heeschen (W.) (1966). - Ein Verfahren zur elektronischen Zählung von Milchzellen. Milchwissenschaft, 31, 93-98.

- Zeidler (H.), Tolle (A.) und HeEschen (W.) (1968 a). - Verbesserte Präparationstechnik zur elektronischen Bestimmung des Zellgehaltes in Milch. Mitchwissenschaft, 23, 564-68.

- Zeidler (H.), Tolle (A.), and Heeschen (W.) (1968 b). - Suitability of direct and indirect cell counting methods for evaluating udder health and milk quality. Arch. Labensmittelhyg., 19, 189-92. Réf. : Dairy Sci. Abstr., 1969, 1026.

\section{Remerciements}

Nous tenons à remercier MM. Mocquot, Plommet, Roguinsky et Ricordeau pour les conseils et critiques qu'ils ont bien voulu nous adresser au cours de ce travail. Nous remercions également M. Tomassone pour l'aide qu'il nous a apportée pour les interprétations statistiques des résultats. 